\title{
METHODOLOGY FOR DIRECT REFLECTANCE MEASUREMENT FROM A DRONE: SYSTEM DESCRIPTION, RADIOMETRIC CALIBRATION AND LATEST RESULTS
}

\author{
L. Markelin ${ }^{1, *}$, J. Suomalainen ${ }^{1}$, T. Hakala ${ }^{1}$, R.A. Oliveira ${ }^{1}$, N. Viljanen ${ }^{1}$, R. Näsi ${ }^{1}$, B. Scott ${ }^{2}$, T. Theocharous ${ }^{2}$, C. Greenwell ${ }^{2}$, \\ N. Fox ${ }^{2}$, E. Honkavaara ${ }^{1}$ \\ ${ }^{1}$ Finnish Geospatial Research Institute FGI, National Land Survey of Finland, FI-00521 Helsinki, Finland - \\ (lauri.markelin,juha.suomalainen,teemu.hakala, raquel.alvesdeoliveira,niko.viljanen,roope.nasi,eija.honkavaara)@nls.fi
}

${ }^{2}$ National Physical Laboratory NPL, Teddington TW11-0LW, UK

(barry.scott,theo.theocharous,claire.greenwell,nigel.fox)@npl.co.uk

\section{Commission I, WG I/1}

KEY WORDS: Hyperspectral, drones, reflectance, radiometric calibration, irradiance

\begin{abstract}
:
We study and analyse performance of a system for direct reflectance measurements from a drone. Key instruments of the system are upwards looking irradiance sensor and downwards looking imaging spectrometer. Requirement for both instruments is that they are radiometrically calibrated, the irradiance sensor has to be horizontally stabilized, and the sensors needs to be accurately synchronized. In our system, irradiance measurements are done with FGI Aerial Image Reference System (FGI AIRS), which uses novel optical levelling methodology and can compensate sensor tilting up to $15^{\circ}$. We performed SI-traceable spectral and radiance calibration of FPI hyperspectral camera at the National Physical Laboratory NPL (Teddington, UK). After the calibration, the radiance accuracy of different channels was between $\pm 4 \%$ when evaluated with independent test data. Sensors response to radiance proved to be highly linear and was on average 0.9994 for all channels. The spectral response calibration showed side peaks on several channels that were due to the multiple orders of interference of the FPI and highlighted the importance of accurate calibration. The drone-based direct reflectance measurement system showed promising results with imagery collected over Jokioinen agricultural grass test site, Finland. AIRS-based image- and band wise image adjustment provided homogenous and seamless image mosaics even under varying illumination conditions and under clouds.
\end{abstract}

\section{INTRODUCTION}

Drone-based (or Unmanned Aerial Vehicle UAV-based) remote sensing has evolved rapidly in recent years. Miniaturized multiand hyperspectral imaging sensors are becoming more common as they provide more abundant information of the object compared to traditional RGB cameras. Reflectance is a physically defined object property and therefore often preferred output of the remote sensing data capture to be used in the further processes (Schaepman-Strub et al., 2006). Absolute radiometric calibration of the sensor provides a possibility for physical modelling of the imaging process and enables efficient procedures for reflectance correction.

To process the radiance images to reflectance factors, methods using ground reference reflectance panels are often feasible because the drone pilot is operating the system locally in the mapping area. Typical method is to use two or more reflectance targets on ground and take calibration image before or after the remote sensing flight. Then, assuming that the illumination conditions over targets and over the object being studied are similar, empirical line based approaches can be used (von Bueren et al., 2015). These conditions are not always met in UAV campaigns. For example, campaigns are often carried out in changing illumination conditions with varying cloudiness (Burkart et al., 2017; Hakala et al., 2013); in such case the in situ reflectance panels do not offer a suitable solution. Secondly, in many situations it is also impossible to reliably employ reflectance panels on the ground at the campaign area. For example, in dense forests the illumination conditions are completely different on ground and at treetops, which are most often the target of interest (Nevalainen et al., 2017). Additionally, panels might not be suitable when operating beyond line of sight.

Recent studies have proposed various approaches to compensate for the challenges caused by varying illumination conditions during campaigns. The most promising approaches are an image based block adjustment (Honkavaara et al., 2014) and correction based on measurement of illumination changes with irradiance sensor on ground or on-board UAV (Burkart et al., 2017; Hakala et al., 2013). In the cases when the only changing component during the flight is the sun elevation, linear interpolation of the calibration parameters between the start and end of the campaign has been suggested. Challenge with irradiance sensors on board drone is the requirement for aligning the irradiance spectrometer accurately in vertical direction (Hakala et al., 2018)

In this paper we study a methodology for direct reflectance measurement from a drone, and present system description, sensor laboratory radiometric calibration, and results from campaign over agricultural grass test site.

\section{MATERIALS AND METHODS}

\subsection{Direct reflectance measurement principle from a drone}

Our methodology for direct reflectance measurements from a drone was first introduced in Hakala et al., (2018). Key instruments of the system are upwards looking horizontally levelled irradiance sensor and downwards looking imaging 
spectrometer. The reflectance factor $\mathrm{R}$ is calculated using the following equation:

$$
R=\pi^{L} / E
$$

where $\mathrm{L}$ is at-sensor radiance reflected by the object and recorded with imaging spectrometer, and $\mathrm{E}$ is the irradiance at object. Requirement for both instruments is that they are radiometrically calibrated, the irradiance sensor has to be horizontally stabilized, and the sensors needs to be accurately synchronized. Similar methodology for direct reflectance measurements from a drone, but using point spectrometers only, has been previously presented by MacArthur et al. (2014).

\subsection{D frame format hyperspectral camera}

The FPI camera prototype $2012 \mathrm{~b}$ captures hyperspectral images in the wavelength range of $500-900 \mathrm{~nm}$ with $10-30 \mathrm{~nm}$ full width at half maximum (FWHM) (Honkavaara et al., 2013; Saari et al., 2013). The camera has a CMOSIS CMV4000 RGB image sensor, focal length of $10.9 \mathrm{~mm}$ and $\mathrm{f}$-number of 2.8. The image size is $1024 \times 648$ pixels with a pixel size of $11 \mu \mathrm{m}$. The spectral range of light reaching the sensor is controlled by adjusting the air gap of the Fabry-Pérot interferometer (FPI) filter. The sequence used in this study provided 36 different bands with full data cube acquired in $1.8 \mathrm{~s}$. The entire camera system weighs less than 700 g. Hyperspectral 2D frame camera allow an object to be imaged from different viewing angles, generating overlapping hyperspectral cubes.

The camera manufacturer has calibrated the camera and provided the software for post processing of the raw FPI data to pseudoradiance images. When the integration time is taken into account pseudoradiances are then converted to radiance in units $\mathrm{W} /\left(\mathrm{m}^{2} \mathrm{sr} \mathrm{nm}\right)$. These radiance images, giving radiance values LMan based on camera manufacturers calibration, are the starting point in further analysis in this study.

\subsection{FGI AIRS irradiance and GNSS/IMU system}

Hemispherical spectral irradiance and GNSS/IMU data was measured with the FGI Aerial Image Reference System (FGI AIRS, Figure 1) (Suomalainen et al., 2017). It consists of a ublox NEO-M8T GNSS receiver, a Vectornav VN-200 IMU, irradiance sensors, a Rasberry Pi 3 single-board computer and a 4G modem. FGI AIRS uses optical levelling to compensate tilting of the irradiance sensor up to $15^{\circ}$ and it can be easily paired with any imaging sensor providing output signal of the image capture times.

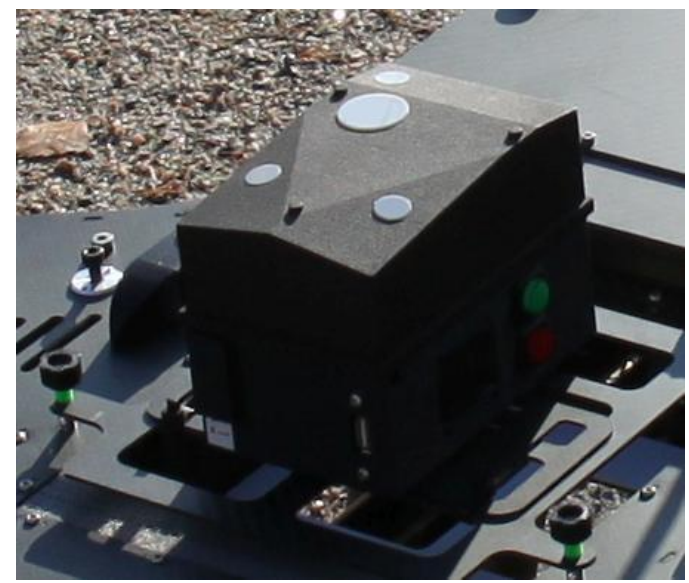

Figure 1. FGI AIRS installed on the top of the drone.

\subsection{Laboratory calibration of the FPI camera}

We performed SI-traceable spectral and radiance calibration of FPI hyperspectral camera at the National Physical Laboratory NPL (Teddington, UK). Details of the laboratory calibration efforts are given in Hakala et al., (2018).

Spectral response calibration of the FPI camera was performed using supercontinuum laser source that was connected to a computer controlled tuneable monochromator. The output from the monochromator was connected to an integrating sphere. The wavelength range of the FPI camera was covered with increments of 1, 2 and $4 \mathrm{~nm}$ steps. The final result of the spectral calibration was full spectral response functions for each FPI camera channel.

The absolute radiance calibration was accomplished by illuminating a reference panel using a lamp with calibrated irradiance (E) and acquiring images of the illuminated panel using the FPI camera. The reflectance factor $(\mathrm{R})$ of the panel and the distance between the lamp and the panel were also measured and thus the radiance of the illuminated panel could be calculated. The FPI camera was placed to an angle of $45^{\circ}$ from the panel at a distance of $1 \mathrm{~m}$. Two lamps were used: a FEL lamp and a Polaron lamp, both of them were calibrated at NPL for irradiance level at $500 \mathrm{~mm}$ distance from the lamp. Combining different camera integration times and lamp distances from the panel, totally 17 different radiance levels were measured.

11 of the radiance data sets were used to calculate traceable radiances $L_{N e w}$ from the FPI camera radiances LMan with a linear calibration model for each band using NPL reference radiance data LNPL_ref:

$$
L_{N P L_{-} \text {ref }}=a \times L_{\text {Man }}+b
$$

where $a$ is multiplicative gain factor to compensate errors in absolute calibration and $b$ is a linear term in radiance units to compensate differences in radiance levels. Six radiance data sets where then used as independent reference to evaluate the final radiance accuracy of the FPI camera.

Linearity of the sensor response to different levels of radiance was evaluated using five radiance levels of Polaron and FEL lamps, all with $10 \mathrm{~ms}$ integration time. Radiances provided by NPL were used as reference and compared to adjusted sensor radiance $\mathrm{L}_{\mathrm{New}}$ based on Equation (2). Linear model was fit between the radiances and $\mathrm{R}^{2}$ values were calculated independently for each band.

\subsection{Test site and drone data sets}

Experimental testing of the direct reflectance measurement with the designed system was carried out in a grass test site located in Jokioinen $\left(68.814309^{\circ} \mathrm{N}, 23.505120^{\circ} \mathrm{E}\right)$, Finland, on $5^{\text {th }}$ September 2017. The flying altitude was $50 \mathrm{~m}$ giving the GSD of $5 \mathrm{~cm}$. The flight speed was $2.3 \mathrm{~m} / \mathrm{s}$ and flight time was 24 minutes. Ground control points (GCPs) were measured in the area using Trimble R10 receiver. Imaging conditions were mainly bright during the campaign, with some varying cloudiness. Irradiance levels during the campaign measured with the FPI camera broad band irradiance sensor is shown in Figure 2 . 


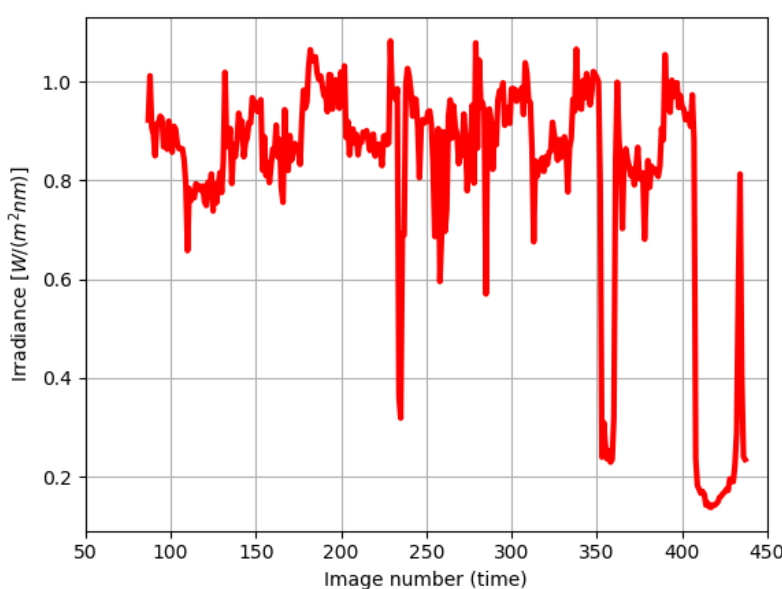

Figure 2. Irradiance during the drone campaign measured with the FPI camera irradiance sensor. Irradiance levels around 1.0 indicates bright illumination conditions.

Two different image mosaics were created from the drone campaign data: one without any radiometric adjustments (ortho) and one where image digital numbers (DNs) where adjusted based on AIRS irradiance data (irrad). One representative FPI image taken under bright illumination conditions was chosen as reference image and its DNs were kept unchanged, and DN's of other images were adjusted relative to reference image. Each FPI band was processed separately, and individual images where combined to mosaic using most nadir method. Mosaicking and image DN adjustments where carried out using the FGI's inhouse developed RadBA-software (Honkavaara et al., 2012, 2013, 2014). The end result of the process is calibrated reflectance image orthomosaic. Details of the geometric processing are given in Oliveira et al., (2018).

\section{RESULTS AND DISCUSSION}

\subsection{Sensor radiometric laboratory calibration}

Spectral responses for bands 10 and 31 are shown in Figure 3 and all bands in Hakala et al., (2018). Many of the FPI 2012b camera bands have side peaks in spectral response, which impact the spectral measurements. Especially bands 25-36 have side peaks at 500-600 $\mathrm{nm}$ wavelength range. The negative values are due to image post-processing. The impurity of the spectral bands, if not cleaned, limits the usability of the sensor in collection of object reflectance data for libraries. If applying spectral libraries in data analysis, the impurity can be accounted for by utilizing the full spectral response curves of each band. In the latest commercial version of the Rikola FPI camera by Senop Ltd. (Oulu, Finland), the FPI side peaks are minimized by not using Bayer pattern on CMOS sensor and dividing spectral data to two different sensors.

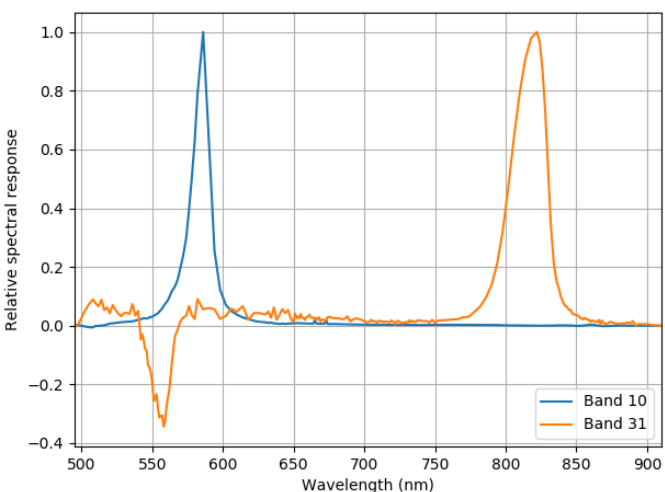

Figure 3. Example of full spectral response functions for bands $10($ centre $w v l=584.43 \mathrm{~nm}, \mathrm{FWHM}=16.64 \mathrm{~nm})$ and $31($ centre $\mathrm{wvl}=816.73 \mathrm{~nm}, \mathrm{FWHM}=27.97 \mathrm{~nm})$. Curves are scaled so that the maximum value is 1 .

Band wise linear parameters $a$ and $b$ to adjust LMan to SI-traceable $\mathrm{L}_{\text {New }}$ were calculated using 11 radiance data sets with equation (2). Accuracy of these parameters was tested with six independent radiance data sets. New adjusted radiance spectra of six independent test cases $\mathrm{L}_{\mathrm{New}}$, reference radiance $\mathrm{L}_{\mathrm{NPL}}$ ref and percentage difference to reference radiance are shown in Figure 4. When comparing the new adjusted radiance to the independent reference, differences were smaller than $4 \%$, and in most cases $<3 \%$.
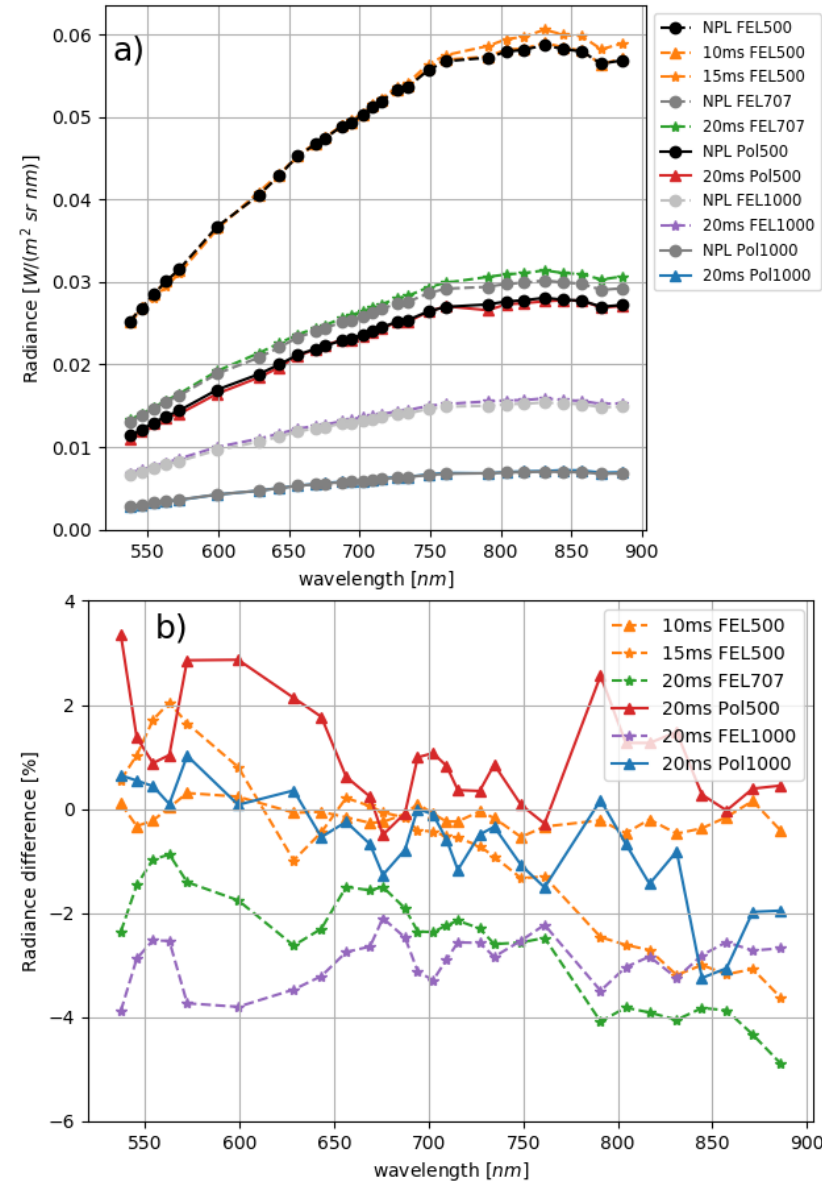

Figure 4. a) New adjusted radiance spectra $\mathrm{L}_{\mathrm{New}}$ (FEL500, FEL707, FEL1000, Pol500 and Pol1000) and NPL reference radiance LNPL_ref (NPL) b) percentage difference to NPL reference LNPL_ref for six independent data sets. 
Radiometric response of all stable bands of the FPI sensor proved to be linear with average $\mathrm{R}^{2}$ of 0.9994 . $\mathrm{R}^{2}$ of best bands (19 and 20) was 1.0000 , and worst two were bands 36 and 29 with $R^{2}$ of 0.9976 and 0.9985 respectively. Example linearity plot for band 27 is shown in Figure 5.

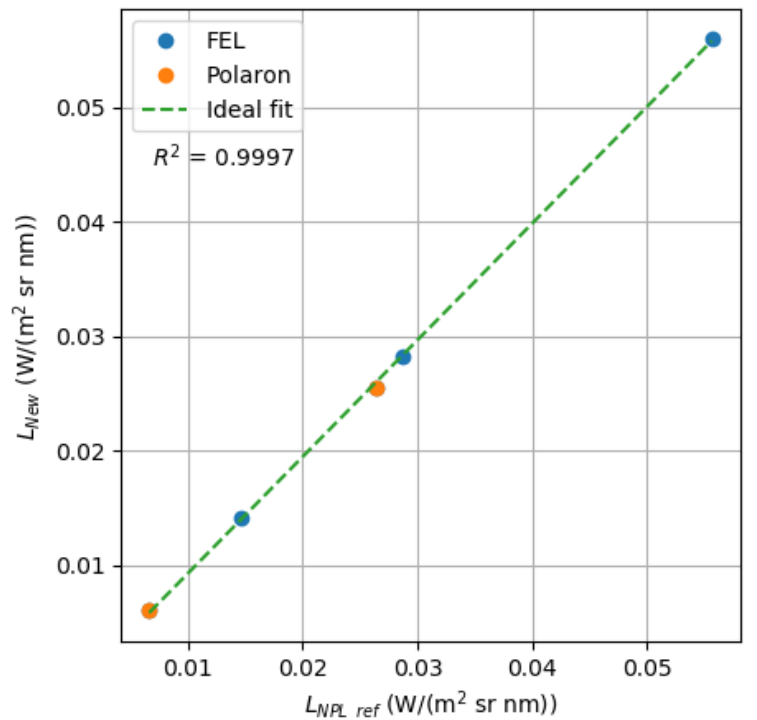

Figure 5. Linearity plot for band 27 (centre wavelength $=748.81$ $\mathrm{nm})$.

\subsection{Drone campaign}

Ortho mosaic without any image adjustments (ortho) showed that clouds shadowed the test area during several image captures (Figure 6a). Radiometric processing using on board AIRS irradiance for image- and band wise adjustment was able to compensate varying illumination conditions resulting homogenous image mosaic (Figure 6b). Examples of false colour image mosaics of both processes, ortho and irrad, are shown in Figure 6.

FGI AIRS was able to provide high quality levelled irradiance measurements for each image acquisition time. Examples of AIRS spectral irradiance for images collected under bright and cloudy conditions are shown in Figure 7. Using FGI AIRS overcomes most of the challenges faced by other studies using either on ground or on board irradiance sensors for correcting drone imagery (Burkart et al., 2017; Hakala et al., 2018, 2013; Miyoshi et al., 2018). Performance, accuracy and quality of the FGI AIRS irradiance needs to be validated in future studies, as well as comprehensive SI-traceable radiometric calibration.

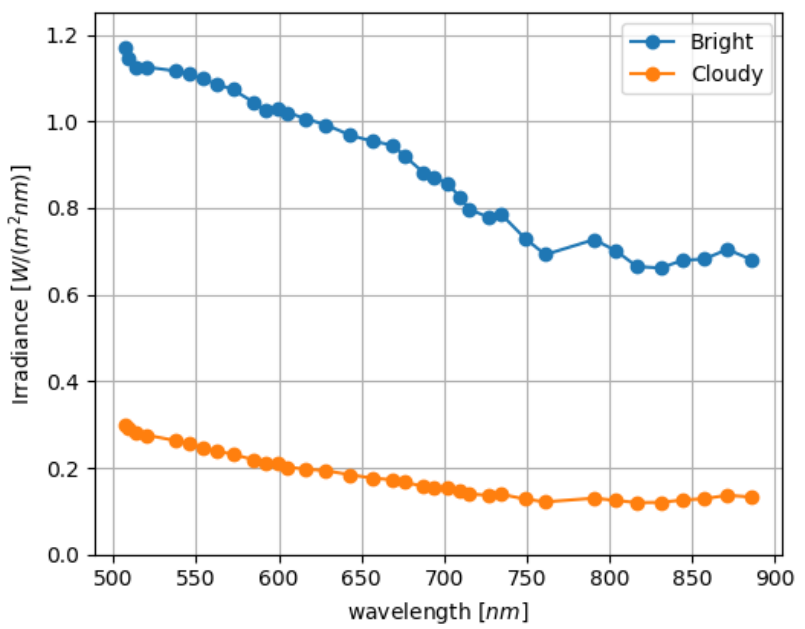

Figure 7. Spectral irradiance measured with FGI AIRS for data cubes collected in bright and cloudy illumination conditions. Line markers show centre wavelength for FPI bands.

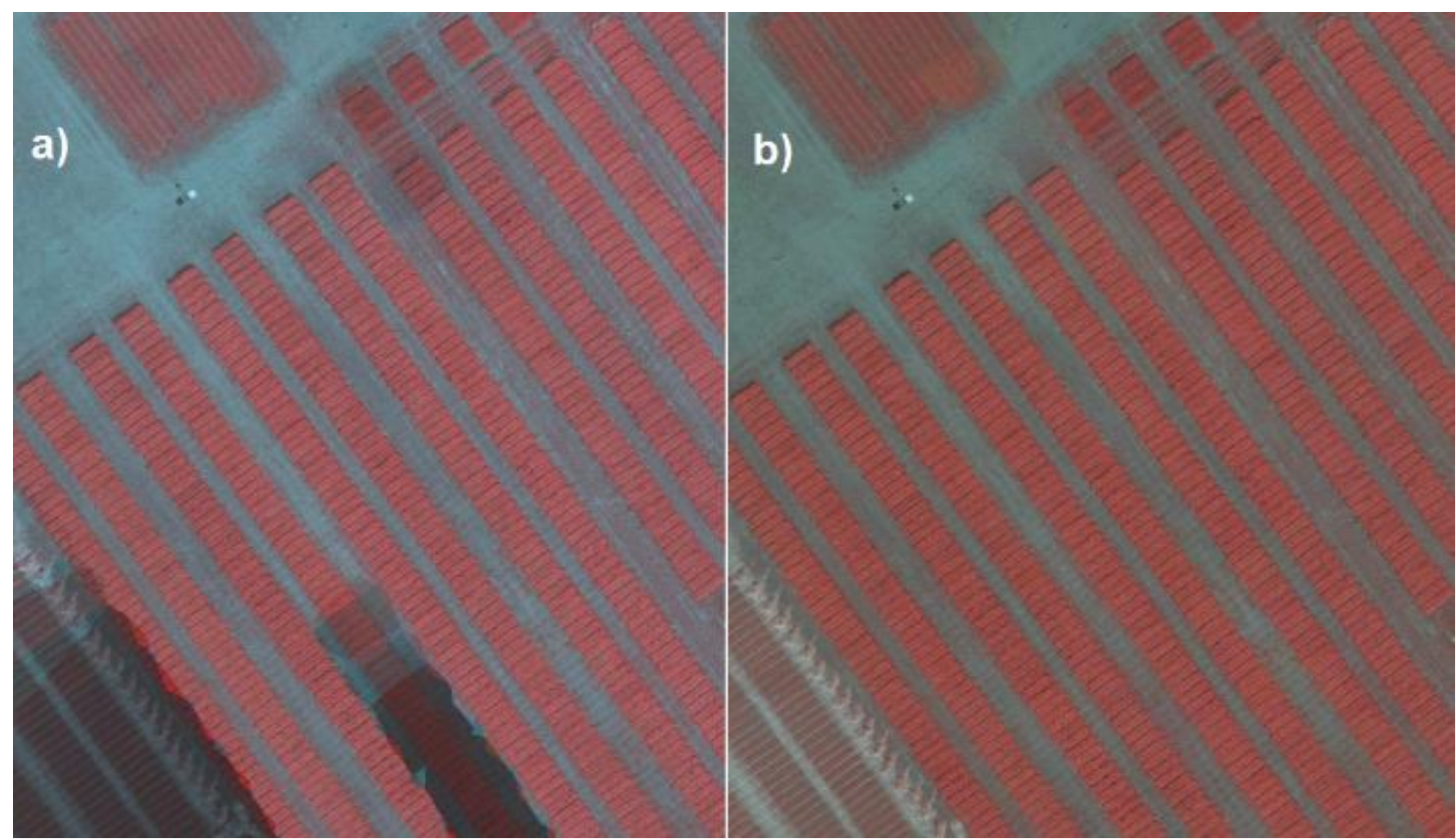

Figure 6. Example false colour reflectance image mosaic of the test area. a) plain ortho, b) with image- and band-wise AIRS irradiance correction. 


\section{CONCLUSIONS}

We presented the first study on direct reflectance measurement using a UAV-based calibrated imaging 2D hyperspectral camera and a novel, calibrated and optically levelled irradiance spectrometer. Our drone-based direct reflectance measurement system has been tested over various test sites and has proved to be highly operational. The proposed approach is extremely attractive, as it simplifies the field operations, and it is suitable for operations in varying illumination conditions, in densely vegetated areas (forests) and beyond line of sight. When calculating the reflectance as the ratio of the incident and reflected radiance based on the imager and the irradiance spectrometer, the radiometric calibration of the two instruments becomes crucial task.

Accurate reflectance mosaics will improve the usability of hyperspectral drone images in various applications such as individual tree detection and recognition (Nevalainen et al., 2017), forest health and pest insect detection (Näsi et al., 2015, 2018a), precision agriculture (Honkavaara et al., 2013; Näsi et al., 2018b), and water quality monitoring (Honkavaara et al., 2014).

\section{ACKNOWLEDGEMENTS}

The research leading to these results was funded through the Metrology for Earth Observation and Climate project (MetEOC2), grant number ENV55 within the European Metrology Research Programme (EMRP). The EMRP is jointly funded by the EMRP participating countries within EURAMET and the European Union. Furthermore, this study was also supported by the Academy of Finland, grant No. 305994.

\section{REFERENCES}

Burkart, A., Hecht, V.L., Kraska, T., Rascher, U., 2017. Phenological analysis of unmanned aerial vehicle based time series of barley imagery with high temporal resolution. Precision Agriculture, Vol. 19(1), pp. 134-146. https://doi.org/10.1007/s11119-017-9504-y

Hakala, T., Honkavaara, E., Saari, H., Mäkynen, J., Kaivosoja, J., Pesonen, L., Pölönen, I., 2013. Spectral imaging from UAVs under varying illumination conditions. In: International Archives of the Photogrammetry, Remote Sensing and Spatial Information Sciences, Vol. XL-1/W2, pp. 189-194. https://doi.org/10.5194/isprsarchives-XL-1-W2-189-2013

Hakala, T., Markelin, L., Honkavaara, E., Scott, B., Theocharous, T., Nevalainen, O., Näsi, R., Suomalainen, J., Viljanen, N., Greenwell, C., Fox, N., 2018. Direct Reflectance Measurements from Drones: Sensor Absolute Radiometric Calibration and System Tests for Forest Reflectance Characterization. Sensors, Vol. 18(5), 1417. https://doi.org/10.3390/s18051417

Honkavaara, E., Hakala, T., Markelin, L., Jaakkola, A., Saari, H., Ojanen, H., Pölönen, I., Tuominen, S., Näsi, R., Rosnell, T., Viljanen, N., 2014. Autonomous hyperspectral UAS photogrammetry for environmental monitoring applications. In: International Archives of the Photogrammetry, Remote Sensing and Spatial Information Sciences, Vol. XL-1, pp. 155-159. https://doi.org/10.5194/isprsarchives-XL-1-155-2014

Honkavaara, E., Hakala, T., Markelin, L., Rosnell, T., Saari, H., Mäkynen, J., 2012. A Process for Radiometric Correction of UAV Image Blocks. Photogrammetrie - Fernerkundung -
Geoinformation, Vol. 2012(2), pp. 115-127. https://doi.org/10.1127/1432-8364/2012/0106

Honkavaara, Eija, Markelin, L., Hakala, T., Peltoniemi, J., 2014. The Metrology of Directional, Spectral Reflectance Factor Measurements Based on Area Format Imaging by UAVs. Photogrammetrie - Fernerkundung - Geoinformation, Vol. 2014(3), pp. 175-188. https://doi.org/10.1127/1432$8364 / 2014 / 0218$

Honkavaara, E., Saari, H., Kaivosoja, J., Pölönen, I., Hakala, T., Litkey, P., Mäkynen, J., Pesonen, L., 2013. Processing and Assessment of Spectrometric, Stereoscopic Imagery Collected Using a Lightweight UAV Spectral Camera for Precision Agriculture. Remote Sensing, Vol. 5(10), pp. 5006-5039. https://doi.org/10.3390/rs5105006

MacArthur, A., Robinson, I., Rossini, M., Davis, N., MacDonald, K., 2014. A dual-field-of-view spectrometer system for reflectance and fluorescence measurements (Piccolo Doppio) and correction of etaloning. In: Proceedings of the Fifth International Workshop on Remote Sensing of Vegetation Fluorescence, European Space Agency, Paris - France, 22-24 April 2014.

Miyoshi, G.T., Imai, N.N., Tommaselli, A.M.G., Honkavaara, E., Näsi, R., Moriya, É.A.S., 2018. Radiometric block adjustment of hyperspectral image blocks in the Brazilian environment. International Journal of Remote Sensing, Vol. 39, 1-21. https://doi.org/10.1080/01431161.2018.1425570

Näsi, R., Honkavaara, E., Blomqvist, M., LyytikäinenSaarenmaa, P., Hakala, T., Viljanen, N., Kantola, T., Holopainen, M., 2018a. Remote sensing of bark beetle damage in urban forests at individual tree level using a novel hyperspectral camera from UAV and aircraft. Urban Forestry \& Urban Greening, Vol. 30, pp. 72-83. https://doi.org/10.1016/j.ufug.2018.01.010

Näsi, R., Honkavaara, E., Lyytikäinen-Saarenmaa, P., Blomqvist, M., Litkey, P., Hakala, T., Viljanen, N., Kantola, T., Tanhuanpää, T., Holopainen, M., 2015. Using UAV-Based Photogrammetry and Hyperspectral Imaging for Mapping Bark Beetle Damage at Tree-Level. Remote Sensing, Vol. 7, pp. 15467-15493. https://doi.org/10.3390/rs71115467

Näsi, R., Viljanen, N., Kaivosoja, J., Alhonoja, K., Markelin, L., Hakala, T., Honkavaara, E., 2018b. Estimating Biomass and Nitrogen Amount of Barley and Grass Using UAV and Aircraft Based Spectral and Photogrammetric 3D Features. Remote Sensing, Vol. 10(7), 1082. https://doi.org/10.3390/rs10071082

Nevalainen, O., Honkavaara, E., Tuominen, S., Viljanen, N., Hakala, T., Yu, X., Hyyppä, J., Saari, H., Pölönen, I., Imai, N., Tommaselli, A., 2017. Individual Tree Detection and Classification with UAV-Based Photogrammetric Point Clouds and Hyperspectral Imaging. Remote Sensing Vol. 9(3), 185. https://doi.org/10.3390/rs9030185

Oliveira, R.A., Khoramshahi, E., Suomalainen, J., Hakala, T., Viljanen, N., Honkavaara, E., 2018. Real-time and post processed georeferencing for hyperspectral drone remote sensing. In: International Archives of the Photogrammetry, Remote Sensing and Spatial Information Sciences, Vol. XLII-2, pp. 789-795. https://doi.org/10.5194/isprs-archives-XLII-2-7892018 
Saari, H., Pölönen, I., Salo, H., Honkavaara, E., Hakala, T., Holmlund, C., Mäkynen, J., Mannila, R., Antila, T., Akujärvi, A., 2013. Miniaturized hyperspectral imager calibration and UAV flight campaigns. In: Proc. SPIE 8889, Sensors, Systems, and Next-Generation Satellites XVII, 888910 (24 October 2013). https://doi.org/10.1117/12.2028972

Schaepman-Strub, G., Schaepman, M.E., Painter, T.H., Dangel, S., Martonchik, J.V., 2006. Reflectance quantities in optical remote sensing - definitions and case studies. Remote Sensing of Environment Vol. 103(1), pp. 27-42. https://doi.org/10.1016/j.rse.2006.03.002

Suomalainen, J., Hakala, T., Honkavaara, E., 2017. Measuring incident irradiance on-board an unstable UAV platform - First results on virtual horizontalization of multiangle measurement. In: Online Proceedings of the International Conference on Unmanned Aerial Vehicles in Geomatics, ISPRS, Bonn, Germany, 4-7 September, 2017.

von Bueren, S.K., Burkart, A., Hueni, A., Rascher, U., Tuohy, M.P., Yule, I.J., 2015. Deploying four optical UAV-based sensors over grassland: challenges and limitations. Biogeosciences, Vol. 12, pp. 163-175. https://doi.org/10.5194/bg-12-163-2015 